\title{
Matteucci and inverse Wiedemann effects in amorphous wires with enhanced circumferential domains
}

\author{
J. J. Freijo, ${ }^{\text {a) }}$ M. Vázquez, and A. Hernando \\ Instituto de Magnetismo Aplicado, Laboratorio "Salvador Velayos"' P.O. Box 155 Las Rozas, \\ Madrid 28230, Spain \\ A. Méndez and V. R. Ramanan \\ ABB Electric Systems Technology Institute, 1021 Main Campus Drive, Raleigh, North Carolina 27606
}

\begin{abstract}
Nonmagnetostrictive $\mathrm{CoFeSiB}$ alloy wires were subjected to cold drawing processing followed by annealing under tension, in order to induce the formation of circular magnetic anisotropy. The axial $\left(M_{Z}-H_{Z}\right)$ and circular $\left(M_{\phi}-H_{\phi}\right)$ hysteresis loops indicate the existence of induced transverse anisotropy, with the magnitude of the anisotropy field being dependent on the processing conditions, while circular loops are quasibistable for samples processed under stress. Very peculiar hysteresis loops are obtained for the Matteucci (MI, $M_{\phi}-H_{Z}$ ) and inverse Wiedemann (IWE, $M_{Z}-H_{\phi}$ ) effects corresponding to those samples exhibiting the mentioned circular bistability. The circular shape of the MI loop is interpreted as produced by reversible magnetization rotation within circumferential domain. The effective hysteresis arises from the existence of a helical distribution of anisotropy in the transition region between the inner axial domain and the external shell. IWE loop confirms the presence of this helicity. (C) 1999 American Institute of Physics. [S0021-8979(99)54408-3]
\end{abstract}

\section{INTRODUCTION}

Amorphous wires fabricated by in-rotating-waterquenching technique ${ }^{1}$ exhibit quite outstanding magnetic properties, that make them very useful for applications as sensing elements in various sensor devices. ${ }^{2,3}$ This is the particular case of wires exhibiting the so-called bistable magnetic behavior, characterized by a single and large Barkhausen jump between two stable remanence states. This magnetic characteristic is spontaneously observed in highly magnetostrictive amorphous wires ${ }^{4}$ and in nearly nonmagnetostrictive Co-based wires after proper processing.

The domain structure of the as-cast Co-based wires is considered as being formed by an inner cylindrical core where the magnetization is axially oriented and an external core with a circular easy axis. Direct observation by Kerr effect technique has shown that, in the as-cast state, the circular outer shell is divided into magnetic domains forming bamboo-like structures. ${ }^{5}$ The aim of the present work is to study both the magnetization process and the domain structure for the case where bistability occurs between two remanences of the circular magnetization process.

\section{EXPERIMENT}

Nonmagnetostrictive $\left(\mathrm{Co}_{96} \mathrm{Fe}_{4}\right)_{72.5} \mathrm{Si}_{12.5} \mathrm{~B}_{15}$ alloy wires have been investigated after having been submitted to the process of cold drawing (reduction of diameter in discrete steps from the as-cast 130 to 100 and $50 \mu \mathrm{m}$ ) and subsequent tension annealing (up to $400 \mathrm{MPa}$ tensile stress at $450^{\circ} \mathrm{C}$ for 15 s) so transverse magnetic anisotropy was induced. Samples after these treatments have been kindly supplied by UNITIKA.

\footnotetext{
${ }^{a}$ Author to whom correspondence should be addressed; electronic mail: freijo@fenix.ima.csic.es
}

The axial $\left(M_{Z}-H_{Z}\right)$ hysteresis loops were measured with a conventional induction technique hysteresis loop tracer. A long solenoid driven by an $80 \mathrm{~Hz}$ triangular signal creates the magnetic axial field $H_{Z}$. Changes in the longitudinal component of the magnetization induce a voltage in a 1000 turns pick-up coil wound around the wire. A second compensating coil is connected in series opposition to cancel the contribution of the $H$ field. The value of the magnetization is obtained by integration of the pick-up signal, and is plotted versus the corresponding $H_{Z}$ field. 30 -cm-long samples were characterizedin the present work. The axial direction of the wires was placed perpendicular to the earth magnetic field, and the solenoid/pick-up coils arrangement was magnetically shielded with a concentric $\mu$-metal cylinder.

The circular magnetization versus circular magnetic field hysteresis curves $\left(M_{\phi}-H_{\phi}\right)$ were also obtained. The circular applied field is produced by a current $I$ flowing through the wire. The generated magnetic field was not homogeneous and increases radially according to

$$
H_{\phi}=\frac{I r}{2 \pi R^{2}},
$$

where $r$ and $R$ are the radial coordinate and the radius, respectively. In order to pick up the magnetization changes no winding can be used and the longitudinal section of the wire itself must be used as a secondary. The measurement method has been fully described elsewhere. ${ }^{6}$ A triangular wave signal, having a frequency of $80 \mathrm{~Hz}$ and maximum amplitude of $20 \mathrm{~mA}$, has been used to drive the wire, which is too small to produce a significant change in the temperature of the wire.

Cross loops, Matteucci $\left(M_{\phi}-H_{Z}\right)$ and inverse Wiedemann $\left(M_{Z}-H_{\phi}\right)$ effects, have been obtained by using a combination of the inductive methods described earlier. 

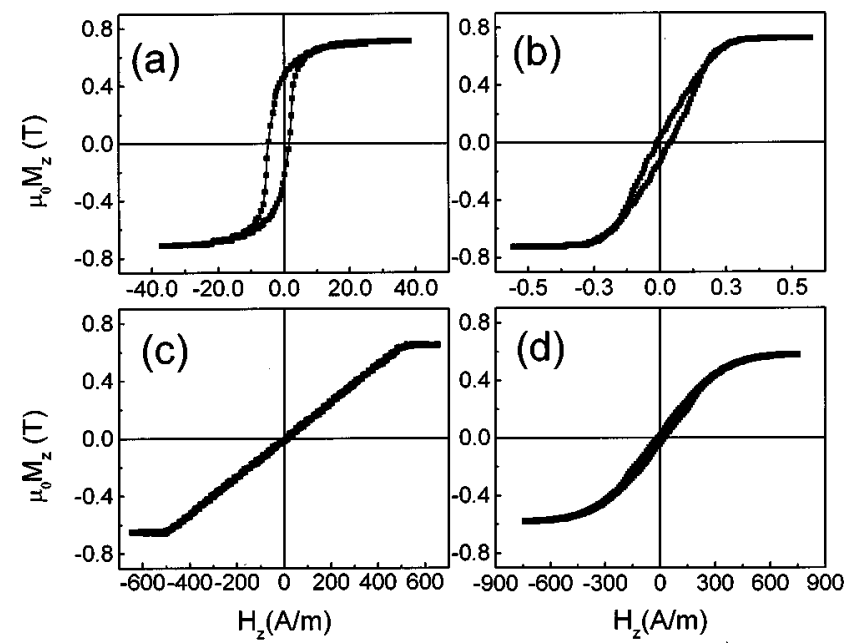

FIG. 1. Axial $\left(M_{Z}-H_{Z}\right)$ hysteresis loops of a $130 \mu \mathrm{m}$ sample in (a) the as-cast state and annealed under a tension of (b) $20 \mathrm{MPa}$, (c) $400 \mathrm{MPa}$. Loop (d) corresponds to a $100-\mu \mathrm{m}$-diam wire in the as-drawn state.

\section{RESULTS AND DISCUSSION}

In Fig. 1, longitudinal hysteresis loops of amorphous wires studied are displayed. The saturation magnetization in all the cases is found to be about $0.72 \mathrm{~T}$. The as-cast 130 $\mu$ m-diam wire [Fig. 1 (a)] approaches saturation at a field of $24 \mathrm{~A} / \mathrm{m}$ and the normalized remanence is $\left(M_{r} / M_{s}\right)=0.55$. The tension annealing process induces transverse anisotropy as can be deduced from Figs. 1(b) and 1(c). When increasing the annealing tension, the induced anisotropy rises and the shape of the loops changes gradually towards a linear nonhysteretic loop typical of coherent rotation when a magnetic field is applied along the hard axis direction.

The hysteresis loop for the as-drawn $100 \mu \mathrm{m}$ sample is displayed in Fig. 1(d). It shows a saturation field around one order of magnitude above that of the as-cast wire. This increase of the magnetic anisotropy is originated during the cold drawn process when multiaxial stresses deform the wires to reduce the sectional area and remain in them. In the as-drawn $50-\mu \mathrm{m}$-diam sample, the successive steps of cold drawing originated higher residual stresses in the same way, giving rise to a higher saturation field. In all the studied wires, the dominant effect of the annealing process under tension of $20 \mathrm{MPa}$ is to remove the high stress present in the as-drawn wire, while annealing under larger stresses induces mainly a circumferential anisotropy.

Numerical values of the transverse anisotropy in these wires can be calculated from the hysteresis loops through the magnetization work, assuming uniaxial anisotropy and magnetization rotation process. The results are displayed in Fig. 2. Induced transversal magnetic anisotropy is shown to be a linear function of the annealing tension and quite independent of the diameter of the samples.

The $\left(M_{\phi}-H_{\phi}\right)$ hysteresis curves of the $130-\mu$ m-diam wire are displayed in Fig. 3. The magnetization has been plotted versus the values of the magnetic field at the surface of the wire. The as-cast wire presents a loop with values of the coercivity and remanence similar to those corresponding to the axial hysteresis loop. After tension annealing the shape

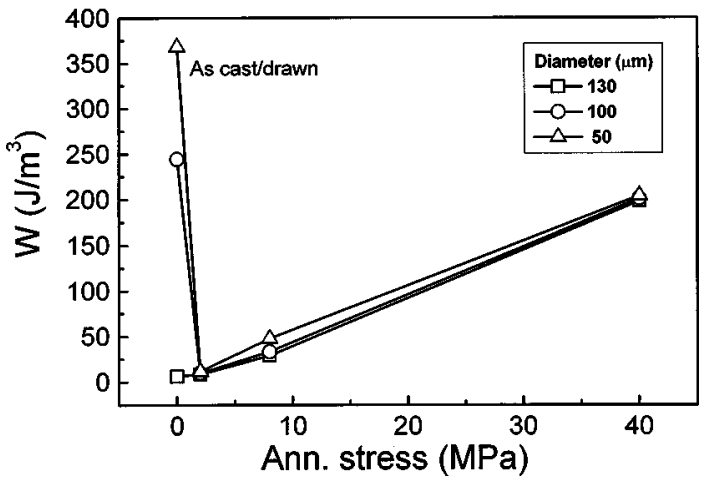

FIG. 2. Induced transverse anisotropy as a function of the tensile stress applied during the thermal treatment.

of the loop changes to a square one, typical for an almost easy axis magnetization process, due to the circular anisotropy induced by the thermal treatment under tensions. The value of the circular coercive field $\left(H_{C \phi}\right)$ is an increasing function of the annealing tension (i.e., the induced anisotropy). Besides, in all the cases, $H_{C \phi}$ is one order of magnitude smaller than the anisotropy field obtained from the axial loops, which indicates that the reversal of the circular magnetization is produced by a domain wall nucleationpropagation process. The value of the normalized remanence equals practically unity in all the thermally treated wires. From these data we can conclude that the outer shell consists of a single domain along the entire length of the wire in which the magnetization has circular orientation. This result contrasts with the bamboo-like domain structures observed in the as-cast wires, ${ }^{5}$ where the stresses frozen during the rapid quenching process yield to a radial component of the magnetization. Thus, minimization of the magnetostatic energy corresponding to the surface poles makes favorable the mentioned domain structure. On the contrary, in the case of the stress annealed samples, the induced anisotropy orientates the magnetization in the circular direction producing no magnetic poles at the surface.

The measurements of the circular magnetic loops for the 100 and $50-\mu \mathrm{m}$-diam wires show similar results. The asdrawn wires present a high coercivity and saturation field, beyond the maximum available field, owing to the stress created in the wire during the cool drawing process. For all the diameters studied, the annealing has a similar effect on the

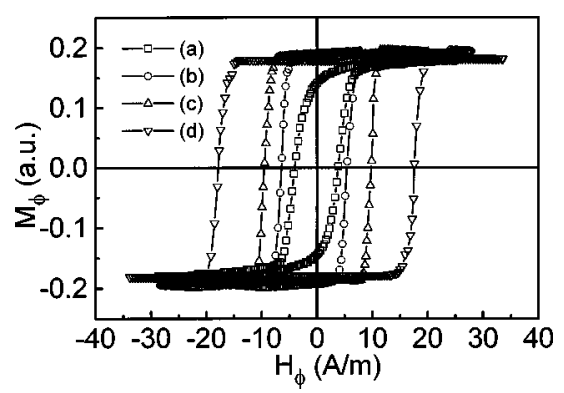

FIG. 3. Circular $\left(M_{\phi}-H_{\phi}\right)$ hysteresis loops for a 130 - $\mu$ m-diam sample in (a) the as-cast state and annealed under a tension of (b) 20, (c) 80, (d) 400 $\mathrm{MPa}$. 

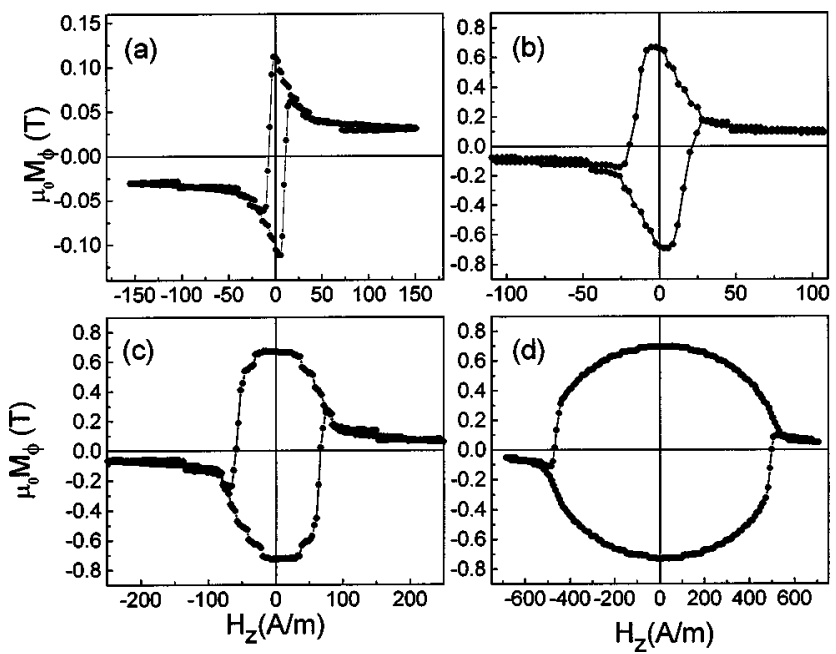

FIG. 4. Mateucci $\left(M_{\phi}-H_{Z}\right)$ hysteresis loops of a $130 \mu \mathrm{m}$ sample in the as-cast state.

circular magnetization: It induces a circular anisotropy that squares the shape of the loops being the coercive field an increasing function of the annealing tension.

The Matteucci $\left(M_{\phi}-H_{Z}\right)$ loops provide a deeper insight into the magnetization process of the studied wires. The results for the $130 \mu \mathrm{m}$ samples are shown in Fig. 4. In the three thermally treated wires under tension, the value of remanent magnetization is very close to the saturation magnetization, in agreement with the results obtained for the circular loops. When an axial magnetic field is applied, the magnetization is tilted towards the axial direction following a rotation process. However, for a critical value of the applied axial field, an irreversible jump occurs which reverses the direction of the circular component of the magnetization; consequently an effective hysteresis appears. The reversal of the circular magnetization under the action of an axial field evidences a helical distribution of anisotropy that links the axial and circular directions. Thus, when the axial field reaches the critical value, the axially oriented magnetization of the inner core reverts, causing the reversal of the circular magnetization. This helicity is expected to be originated during the annealing process in the transition region between the core and the outer shell characterized by a helical distribution of the magnetization.

Inverse Wiedemann loops for the stress annealed 130$\mu \mathrm{m}$-diam samples are shown in Fig. 5. Large Barkhausen

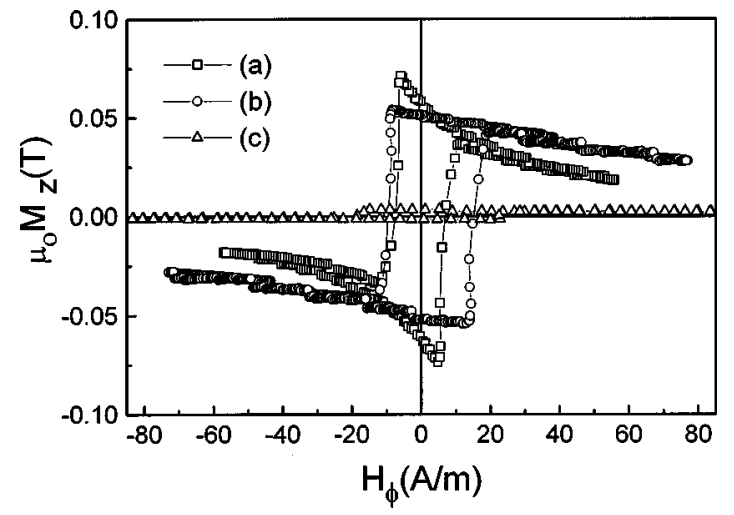

FIG. 5. Inverse Wiedemann $\left(M_{Z}-H_{\phi}\right)$ hysteresis loops of a $130 \mu \mathrm{m}$ sample annealed under (a) 20, (b) 80, (c) $400 \mathrm{MPa}$.

jumps are observed at the coercive field of the circular loops. This confirms the existence of the aforementioned helical anisoropy. The jumps correspond to the reversal of the magnetization in the core, caused by the inversion of the outer shell.

\section{CONCLUSIONS}

The experimental results shown in this article point out that the control of the anisotropy through stress annealing allows us control of the magnetization process. In the samples studied, the stress annealing process induces a noticeable transverse anisotropy that produces circular bistability. The Mateucci and the inverse Wiedemann effect loops are proven to be powerful tools for the study of the magnetization process of this kind of material. In particular they evidenced a helical distribution of anisotropies that links the axially oriented core with the circularly oriented outer domain.

${ }^{1}$ I. Ogasawara and S. Ueno, IEEE Trans. Magn. 31, 1219 (1995).

${ }^{2}$ F. B. Humphrey, Mater. Sci. Eng., A 179/180, 66 (1994).

${ }^{3}$ M. Vázquez, M. Knobel, M. L. Sánchez, R. Valenzuela, and A. P. Zhukov, Sens. Actuators A 59, 20 (1997).

${ }^{4}$ F. B. Humphrey et al., in Re-entrant Magnetic Flux Reversal in Amorphous Wires, edited by A. Hernando et al. (Elsevier, Amsterdam, 1987), pp. 110-116.

${ }^{5}$ J. Yamasaki, J. Magn. Soc. Jpn. 16, 14 (1992).

${ }^{6}$ A. Hernando and J. M. Barandiarán, J. Phys. D: Appl. Phys. 11, 1539 (1978). 